\title{
Functional Effects of the Adams Procedure: A Retrospective Intervention Study
}

\author{
Thomas J. M. Kootstra, BSc ${ }^{1} \quad$ Margriet H. van Doesburg, MD, $\mathrm{PhD}^{1} \quad$ Arnold H. Schuurman, MD, $\mathrm{PhD}^{1}$ \\ 1 Department of Plastic Surgery, Universitair Medisch Centrum \\ Utrecht, Universiteit Utrecht, Utrecht, The Netherlands \\ Address for correspondence Arnold H. Schuurman, MD, PhD, \\ Department of Plastic Surgery, Universitair Medisch Centrum Utrecht, \\ Universiteit Utrecht, Heidelberglaan 100, 3584 CX, Utrecht, \\ J Wrist Surg 2018;7:331-335. \\ The Netherlands (e-mail: a.schuurman@umcutrecht.nl).
}

\begin{abstract}
Keywords

- function

- long term

- Adams procedure

- DRUJ

- reconstruction

- DASH

- PRWHE

Background Injury to the distal radioulnar joint (DRUJ) causes swelling and ulnarsided wrist pain. The Adams procedure stabilizes the DRUJ and shows promising shortterm results.

Purpose We studied the long-term functional outcome in patients who underwent the Adams procedure. We also tested the null hypothesis that there would be no difference in range of motion (ROM) and grip strength between the operated wrist compared to the unaffected side.

Patients and Methods We identified 74 consecutive patients that were operated from March 2005 to February 2014. Twenty-three patients responded to our invitation and underwent clinical follow-up. Three patients had a re-do of the Adams procedure after presenting with postoperative DRUJ instability, one was excluded due to additional wrist surgery not related to the initial procedure. Wrist functionality in the remaining 22 patients was examined by measuring ROM in all planes and grip strength of the operated and non-operated hand. Functional outcome was measured by asking patients to complete the Disabilities of the Arm, Shoulder, and Hand (DASH) outcome measure and the Patient-Rated Wrist and Hand Evaluation (PRWHE).

Results After a mean follow-up time of 5.0 years (standard deviation [SD] 2.0), we found that supination, pronation, and grip strength were significantly decreased in the operated wrist. Postoperative DASH and PRWHE-scores averaged 13.1 (SD 18.6) and 20.3 (SD 15.1), respectively.

Conclusion The Adams procedure affects, but largely restores pronation and supination, and clinical examination, DASH, and PRWHE scores indicate that the Adams procedure leaves patients with upper extremity functionality comparable to a healthy population.

Level of Evidence Level IV.
\end{abstract}

The distal radioulnar joint (DRUJ) is part of a complex articulation in the forearm that provides the distal weightbearing link between the radius and ulna ${ }^{1,2}$ and a pivot for pronation and supination. ${ }^{3}$ Following a fall on the outstretched hand, ${ }^{4,5}$ injury to the DRUJ can occur in isolation or with a fracture of the distal radius ${ }^{6-8}$ and causes swelling and ulnar-sided wrist pain aggravated by forearm motion. ${ }^{9}$ Pain-at-rest and swelling typically improve, but pain with use continues and may become associated with wrist instability including weakness and a joint clunk. ${ }^{9}$

The DRUJ relies heavily on soft tissue (i.e., ligament, muscle) support for stability, the triangular fibrocartilage received

June 14, 2017

accepted after revision

May 10, 2018

published online

June 13, 2018
Copyright $\odot 2018$ by Thieme Medical Publishers, Inc., 333 Seventh Avenue, New York, NY 10001, USA. Tel: +1(212) 584-4662. 
complex (TFCC) being the most important for maintaining normal kinematics. 2,10,11 When the TFCC is irreparable because of retraction or primary tissue damage, reconstruction of the volar and dorsal radioulnar ligaments (RULs) at their anatomic origins and insertions offers the best possibility if the biomechanical role of the DRUJ is to be preserved. ${ }^{10,12}$ A technique described in $2000,{ }^{13}$ called the Adams procedure, stabilizes the DRUJ and shows promising short-term results with improvement in pain, grip strength, and function with restoration of joint stability. ${ }^{12}$

This led to the question what the long-term functional outcome is in patients who underwent an Adams procedure. We also tested the null hypothesis that there would be no difference in range of motion (ROM) and grip strength between wrists in patients who underwent the Adams procedure compared to the unaffected side.

\section{Patients and Methods}

\section{Design}

This retrospective study was approved by our institutional review board. All consecutive patients with DRUJ instability who underwent the Adams procedure in our institution between March 2005 and February 2014 were asked to participate in this study. A total of 74 patients underwent the procedure, of whom 31 responded for participation. Inclusion criteria included DRUJ instability with chronic wrist pain and a minimal age of 18 years. Twenty-three of 31 patients eventually responded to the invitation for clinical follow up, one patient had additional wrist surgery not related to the initial operation and was subsequently excluded. This left us with a final cohort of 22 patients (-Table 1). Of the 22 enrolled patients, 9 (41\%) were male, and 13 (59\%) were female. All of the responders were Caucasian. Average age at the time of operation was 35.2 years (standard deviation [SD] 15.2), with an overall mean followup time of 5.0 years (SD 2.0). Average age at the time of the examination was 40.2 years (SD 15.4). The dominant hand

Table 1 Demographics

\begin{tabular}{|l|l|l|}
\hline$n$ & 22 \\
\hline Gender & $\begin{array}{l}9 \text { males (41\%); 13 females } \\
(59 \%)\end{array}$ \\
\hline Dominant hand & 20 right (91\%); 2 left (9\%) \\
\hline Surgery hand & 7 right (32\%); 15 left (68\%) \\
\hline $\begin{array}{l}\text { Surgery on the dominant } \\
\text { side }\end{array}$ & 9 (41\%) \\
\hline & Average \pm SD & Range \\
\hline $\begin{array}{l}\text { Age at time of operation } \\
\text { (years) }\end{array}$ & $35.2 \pm 15.2$ & $(17.3-63.3)$ \\
\hline Follow-up (years) & $5.0 \pm 2.0$ & $(1.7-8.5)$ \\
\hline $\begin{array}{l}\text { Age at time of examination } \\
\text { (years) }\end{array}$ & $40.2 \pm 15.4$ & $(19.5-70.3)$ \\
\hline
\end{tabular}

Abbreviation: SD, standard deviation. was operated on in 9 (41\%) patients. Three patients suffered a distal radius fracture, four patients suffered a distal radius fracture together with an ulnar styloid fracture, and two patients suffered an isolated ulnar styloid fracture. There were two patients who had a preceding ulnar shortening osteotomy; no other preceding operations were noted. After review of the patient records and radiologic reports, four patients with ulnar styloid nonunion were noted, and no patients who had functional limitations due to a radiologically confirmed distal radius deformity or malunion were noted. No patients had DRUJ widening or arthritis.

After obtaining informed consent, the patients' wrist function was examined by measuring ROM and grip strength. We also recorded postoperative disability by asking patients to complete the Dutch language version (DLV) of the Disabilities of the Arm, Shoulder, and Hand (DASH-DLV) ${ }^{14}$ questionnaire, as well as the Patient-Rated Wrist and Hand Evaluation (PRWHE-DLV). ${ }^{15}$ All measurements and assessments were done by a researcher not directly related to patient care.

\section{Outcome Measures}

We measured wrist ROM postoperatively in both wrists. We subsequently compared the ROM of the operated wrist with the ROM of the non-operated wrist. The ROM movements included wrist flexion, extension, ulnar deviation, radial deviation, supination, and pronation. We reported the results as percentages of the healthy contralateral wrist.

Grip strength was determined using the Jamar dynamometer at level two (Sammons Preston, Bollingbrook, IL) and reported absolute values in kilograms (kg). We asked patients to grip the dynamometer three consecutive times, alternating between non-operated and operated hand and calculated an average grip strength for each. Again, we reported the results as percentages of the healthy contralateral wrist.

We measured subjective results using the DASH-DLV score and the PRWHE-DLV score. The DASH-DLV is a standardized questionnaire that reports the patient's own assessment of upper extremity disability and ability to perform certain tasks. It consists of 30 items, each being rated on a 5point scale. The PRWHE-DLV is another standardized questionnaire, designed to evaluate pain and function from the patient's perspective. It consists of three sections (pain, functionality, and cosmetic). Both questionnaires are scored from 0 to 100 , where 0 corresponds with no complaints, and a higher score indicates greater disability.

\section{Surgical Technique}

Reconstruction of the RULs was performed as described by Adams et al. ${ }^{9,13}$ In summary, the TFCC is reconstructed by drilling a tunnel through the radius proximal to the lunate fossa and radial to the articular surface of the sigmoid notch. Another tunnel is drilled obliquely through the ulnar head from the ulnar neck at its subcutaneous border to the fovea of the ulnar head, after which a palmaris longus, toe extensor tendon, or plantaris tendon is harvested and pulled through both tunnels, The two limbs are subsequently pulled taut. 
Functional Effects of the Adams Procedure Kootstra et al. 333

\section{Postoperative Treatment}

All patients had been given above elbow circular cast for 3 weeks followed by removable forearm cast for 3 weeks, combined with hand therapy. Hand therapy lasted for 3 to 6 months.

\section{Statistical Analysis}

Patient demographics were reported using descriptive statistics. Means, SD, and ranges were reported for continuous variables, frequencies, and percentages for categorical variables. We performed bivariate analysis using Student's $t$-test for continuous variables to assess the association between continuous variables and having an Adams procedure. A twosided $P$-value of $<0.05$ was considered statistically significant.

\section{Results}

The senior author (A.H.S.) clinically evaluated the stability of the DRUJ in all patients postoperatively and concluded after an average time of 7.8 months (range: 1.6-40) that all wrists were stable. Patients who returned with wrist instability all did so after DRUJ stability was initially confirmed clinically by the senior author (A.H.S.).

Three patients had additional surgical procedures for recurrent instability. These patients had heard and felt a "snap" in the wrist. Time from the operation to return to the outpatient clinic with these complaints ranged from 7 months to a little over 3 years. Diagnostic wrist magnetic resonance imaging (MRI) confirmed complete detachment of the tendon grafts in all three patients. Which part the tendon grafts had detached from was not recoverable from the MRI reports. However, it is likely that they had done so at either entrance of the radial drill hole due to attenuation of one of the graft limbs, as the angle the graft limbs make is the sharpest there. They had a re-do of the Adams procedure 3 to 10 months after detachment of the tendon graft, after which wrist stability was regained. Twenty-two patients, including the three patients who had a re-do of the Adams procedure, were examined and asked to finish the questionnaires after a mean follow-up of 5.0 years (SD 2.0). After this period, DASHscores averaged 13.1 (SD 18.6), and PRWHE scores averaged 20.3 (SD 17.1, - Table 2).
Table 2 Postoperative DASH and PRWHE scores

\begin{tabular}{|l|l|}
\hline Questionnaire & Average \pm SD (range) \\
\hline DASH & $13.1 \pm 18.6(0-82)$ \\
\hline PRWHE & $20.3 \pm 17.1(2-65)$ \\
\hline
\end{tabular}

Abbreviations: DASH, Disabilities of the Arm, Shoulder, Hand; PRWHE, patient-rated wrist and hand evaluation; SD, standard deviation.

In these 22 patients, we found significant differences in supination and pronation between the operated and nonoperated hand in favor of the healthy wrist ( - Table 3 ). There was no statistically significant difference between the healthy wrist and operated wrist in all other planes of motion. Furthermore, we found a significant difference in postoperative grip strength between the non-operative and operative hand.

\section{Discussion}

The DRUJ is of vital importance if the biomechanical role of the wrist is to be preserved, ${ }^{2}$ and the Adams procedure was designed to conserve its function by reconstructing the volar and dorsal RULs. The main findings of this study were that the Adams procedure yields good long-term results while looking at wrist function and disability, since we found no significant difference in ROM between the healthy wrists and the operated wrists in all planes of motion except supination and pronation. We also found a significant difference in grip strength between the two hands in favor of the healthy hand.

Our findings should be considered in light of a few shortcomings. Due to a low response rate, our sample size might not be large enough to reveal a true difference within the treatment group. Also, the retrospective nature is an obvious drawback. Functional results could only be compared with the contralateral side and not to the preoperative situation. In the future, larger and preferably randomized studies are needed.

Gugger et al reported an average postoperative DASH score of $24.4(3-57)^{16}$ which, compared with our findings

Table 3 Range of motion and grip strength of the wrists

\begin{tabular}{|l|l|l|l|l|}
\hline $\begin{array}{l}\text { Range of motion } \\
\text { (degrees) }\end{array}$ & $\begin{array}{l}\text { Non-operated wrist } \\
\text { average } \pm \text { SD (range) }\end{array}$ & $\begin{array}{l}\text { Operated wrist } \\
\text { average } \pm \text { SD (range) }\end{array}$ & $\begin{array}{l}\text { Operated/non-operated } \\
\text { wrist } \pm \text { SD (range) }\end{array}$ & $p$-Value \\
\hline Flexion & $70.5 \pm 10.3(45-90)$ & $67.0 \pm 6.7(55-80)$ & $97.4 \pm 20.7(70.6-177)$ & 0.18 \\
\hline Extension & $71.8 \pm 7.8(60-85)$ & $71.8 \pm 8.4(55-85)$ & $101 \pm 14.0(70.6-123)$ & 1.00 \\
\hline Ulnar deviation & $34.1 \pm 7.0(25-55)$ & $30.9 \pm 7.0(20-50)$ & $93.0 \pm 23.9(57.1-142)$ & 0.10 \\
\hline Radial deviation & $20.5 \pm 5.5(10-35)$ & $17.7 \pm 4.8(5.0-25)$ & $91.5 \pm 32.1(25.0-150)$ & 0.062 \\
\hline Supination & $80.9 \pm 13.2(45-100)$ & $71.4 \pm 17.1(30-105)$ & $93.0 \pm 25.9(11.8-150)$ & 0.048 \\
\hline Pronation & $82.0 \pm 9.1(60-100)$ & $73.0 \pm 17.9(10-95)$ & $90.2 \pm 25.2(50.0-166)$ & 0.026 \\
\hline Grip strength $(\mathrm{kg})$ & $\begin{array}{l}\text { Non-operated wrist } \\
\text { average } \pm \text { SD (range) }\end{array}$ & $\begin{array}{l}\text { Operated wrist } \\
\text { average } \pm \text { SD (range) }\end{array}$ & $\begin{array}{l}\text { Operated/non-operated } \\
\text { wrist } \pm \text { SD (range) }\end{array}$ & $p$-Value \\
\hline Three time average & $42.7 \pm 14.3(22-68)$ & $38.6 \pm 17.2(9.0-79)$ & $89.7 \pm 22.4(30.2-127)$ & 0.013 \\
\hline
\end{tabular}

Abbreviation: SD, standard deviation. 
(13.1 SD 18.6), exhibits greater disability. The contrast to our findings could be explained by the large number of radius fractures in their cohort. We found no other studies that reported DASH scores in patients who underwent the Adams procedure. Jester et $\mathrm{al}^{17}$ found a mean DASH score of 13 points (SD 15) in a healthy working population, which is comparable to the mean postoperative score of our study population (13.1 SD 18.6).We found no studies that reported PRWHE scores in patients who underwent the Adams procedure.

The range of pronation and supination is arguably the most important indicator of integrity of the DRUJ. ${ }^{18}$ Adams and Lawler aimed for a pronation and supination ROM of at least $85 \% 6$ months postoperatively compared with preoperative measurements. ${ }^{10}$ Gugger et al also compared the operated wrist with the healthy wrist and reported an average postoperative pronation an supination ROM of $89 \%$ with a similar average follow-up time of 5.6 years (2.2-9.2). ${ }^{16}$ Adams and Berger reported average postoperative pronation and supination ROM of $84 \%$ and $80 \%$, respectively, when compared with the healthy side and unchanged flexionextension and radial-ulnar deviation ROM with an average follow-up of 2.2 years (1-4). ${ }^{12}$ The pronation-supination rates reported are lower compared with our findings with 90.2\% (SD 25.2) and 93.0\% (SD 25.9), respectively, and the significant difference in pronation we found may be the result of the position of the reconstructed dorsal RUL during immobilization. ${ }^{2,7}$

Adams and Berger measured an average postoperative grip strength of $85 \%$ of the healthy hand, ${ }^{12}$ which is comparable to our findings with a longer follow-up (89.7\% SD 22.4). While Gugger et al measured an average postoperative grip strength of only $69 \%$ (40-105) compared with the healthy hand. ${ }^{16}$ The difference to our findings could again be explained by the large number of radius fractures in their cohort (almost 80\%).

Henry $^{19}$ described a procedure that also stabilizes the DRUJ and, in contrast to the Adams procedure, replicates the normal anatomy by restoring both the volar and dorsal limbs of the RUL by re-attaching the RUL through intraarticular coronal drill tunnels in the radius. After an average of 51 months postoperatively, he reported better DASH scores $(7 \pm 3)$. However, he did describe worse percentages of contralateral grip strength (79\%), and worse ROM was reported with flexion, extension, supination, and pronation averaging $61 \pm 10,62 \pm 15,74 \pm 7$, and $71 \pm 8$, respectively. The discrepancies found here might be because these measurements were all taken at 6 months postoperatively in contrast to our measurements, which were taken at an average of 5.0 years postoperatively. Brink and Hanneman 20 studied a relatively new technique that stabilizes the DRUJ by pulling a palmaris tendon through drilled canals in the radius and ulna. A central knot prevents the tendon from slipping out of the radius on one end; a tenodesis screw prevents the tendon from slipping out the ulna on the other end. Tension and subsequent stability are thus ensured. After a mean follow-up time of 25 months, they reported worse average QuickDASH and PRWHE scores, averaging 29.6 and 33.6, respectively. The differences to the scores reported in the present study might be explained by the fact that their cohort included only posttraumatic patients. Unfortunately, no other articles describing procedures that restored total anatomic function (in contrast to only the dorsal or volar RUL) were found in relation to patient reported outcomes.

In conclusion, reconstruction of the DRUJ according to Adams affects but largely restores pronation and supination. Furthermore, it yields good results in the long term and compares well to other procedures restoring the DRUJ. Postoperative clinical examination, DASH, and PRWHE scores indicate that the Adams procedure is effective in the elimination of gross instability of the wrist, leaving patients with an upper extremity functionality comparable to that of a healthy working population.

Note

This work was performed at the Division of Plastic Surgery, Universitair Medisch Centrum Utrecht, Universiteit Utrecht, Utrecht, the Netherlands.

\section{Ethical Approval}

This study was approved by the Institutional Review Board.

Funding

None.

Conflict of Interest

None.

\section{References}

1 Fujitani R, Omokawa S, Akahane M, Iida A, Ono H, Tanaka Y. Predictors of distal radioulnar joint instability in distal radius fractures. J Hand Surg Am 2011;36(12):1919-1925

2 Scheker LR, Belliappa PP, Acosta R, German DS. Reconstruction of the dorsal ligament of the triangular fibrocartilage complex. J Hand Surg [Br] 1994;19(03):310-318

3 Bain GI, Pourgiezis N, Roth JH. Surgical approaches to the distal radioulnar joint. Tech Hand Up Extrem Surg 2007;11(01):51-56

4 Atzei A. New trends in arthroscopic management of type 1-B TFCC injuries with DRUJ instability. J Hand Surg Eur Vol 2009;34(05): 582-591

5 Atzei A, Rizzo A, Luchetti R, Fairplay T. Arthroscopic foveal repair of triangular fibrocartilage complex peripheral lesion with distal radioulnar joint instability. Tech Hand Up Extrem Surg 2008;12 (04):226-235

6 Bombaci H, Polat A, Deniz G, Akinci O. The value of plain X-rays in predicting TFCC injury after distal radial fractures. J Hand Surg Eur Vol 2008;33(03):322-326

7 Wijffels M, Brink P, Schipper I. Clinical and non-clinical aspects of distal radioulnar joint instability. Open Orthop J 2012;6:204-210

8 Thomas BP, Sreekanth R. Distal radioulnar joint injuries. Indian J Orthop 2012;46(05):493-504

9 Lawler E, Adams BD. Reconstruction for DRUJ instability. Hand (NY) 2007;2(03):123-126

10 Adams BD, Lawler E. Chronic instability of the distal radioulnar joint. J Am Acad Orthop Surg 2007;15(09):571-575

11 Palmer AK, Werner FW. The triangular fibrocartilage complex of the wrist-anatomy and function. J Hand Surg Am 1981;6(02): 153-162 
12 Adams BD, Berger RA. An anatomic reconstruction of the distal radioulnar ligaments for posttraumatic distal radioulnar joint instability. J Hand Surg Am 2002;27(02):243-251

13 Adams BD. Anatomic Reconstruction of the Distal Radioulnar Ligaments for DRUJ Instability. Tech Hand Up Extrem Surg 2000;4 (03):154-160

14 MacDermid JC, Turgeon T, Richards RS, Beadle M, Roth JH. Patient rating of wrist pain and disability: a reliable and valid measurement tool. J Orthop Trauma 1998;12(08):577-586

15 Hudak PL, Amadio PC, Bombardier C; The Upper Extremity Collaborative Group (UECG). Development of an upper extremity outcome measure: the DASH (disabilities of the arm, shoulder and hand) [corrected]. Am J Ind Med 1996;29(06):602-608

16 Gugger Y, Mühldorfer M, Prommersberger KJ, van Schoonhoven J. [Stabilization of the distal radioulnar joint according to Adams: clinical, and radiological results]. Handchir Mikrochir Plast Chir 2015;47(05):297-303

17 Jester A, Harth A, Germann G. Measuring levels of upper-extremity disability in employed adults using the DASH Questionnaire. J Hand Surg Am 2005;30(05):1074.e11074.e10

18 Cardenas-Montemayor E, Hartl JF, Wolf MB, et al. Subjective and objective results of arthroscopic debridement of ulnar-sided TFCC (Palmer type 1B) lesions with stable distal radio-ulnar joint. Arch Orthop Trauma Surg 2013;133(02):287-293

19 Henry M. Anatomic reconstruction of the radioulnar ligament. Hand (NY) 2012;7(04):413-419

20 Brink PRG, Hannemann PFW. Distal oblique bundle reinforcement for treatment of DRUJ instability. J Wrist Surg 2015;4(03): $221-228$ 